\title{
Carrier Multiplication and Its Reduction by Photodoping in Colloidal InAs Quantum Dots
}

\author{
J. J. H. Pijpers, ${ }^{*}, \dagger$ E. Hendry ${ }^{\dagger}$ M. T. W. Milder, ${ }^{\dagger}$ R. Fanciulli, ${ }^{\dagger}$ J. Savolainen, ${ }^{\dagger}$ J. L. Herek, ${ }^{\dagger}$ \\ D. Vanmaekelbergh, ${ }^{\ddagger}$ S. Ruhman, ${ }^{\S}$ D. Mocatta, ${ }^{\S}$ D. Oron, ${ }^{\S}$ A. Aharoni, ${ }^{\S}$ U. Banin, ${ }^{\S}$ and \\ M. Bonn ${ }^{\dagger}$
}

FOM Institute for Atomic and Molecular Physics (AMOLF), Kruislaan 407, 1098 SJ Amsterdam, The Netherlands, Department of Condensed Matter and Interfaces, Debye Institute, Utrecht University, Princetonplein 5, 3508 TA Utrecht, The Netherlands, and Institute of Chemistry and the Center for Nanoscience and Nanotechnology, the Hebrew University of Jerusalem, Jerusalem 91904, Israel

Received: October 12, 2006; In Final Form: December 12, 2006

\begin{abstract}
Carrier (exciton) multiplication in colloidal InAs/CdSe/ZnSe core-shell quantum dots (QDs) is investigated using terahertz time-domain spectroscopy, time-resolved transient absorption, and quasi-continuous wave excitation spectroscopy. For excitation by high-energy photons ( $\sim 2.7$ times the band gap energy), highly efficient carrier multiplication $(\mathrm{CM})$ results in the appearance of multi-excitons, amounting to $\sim 1.6$ excitons per absorbed photon. Multi-exciton recombination occurs within tens of picoseconds via Auger-type processes. Photodoping (i.e., photoinjection of an exciton) of the QDs prior to excitation results in a reduction of the $\mathrm{CM}$ efficiency to $\sim 1.3$. This exciton-induced reduction of CM efficiency can be explained by the twofold degeneracy of the lowest conduction band energy level. We discuss the implications of our findings for the potential application of InAs QDs as light absorbers in solar cells.
\end{abstract}

\section{Introduction}

Recently, III-V semiconductor quantum dots (QDs) have become available with strong absorption cross sections and bright and stable luminescence. ${ }^{1}$ These particles cover the visible to near-infrared spectral range and have important potential applications in biology and electro-optical devices. ${ }^{2-7}$ Such electro-optic devices rely on the conversion of light into excited electrons and holes (excitons) and vice versa, and knowledge of the processes immediately following photoexcitation is therefore essential for these applications, in addition to the fundamental interest in exciton and charge dynamics in QDs.

As such, there has been much interest recently in exciton dynamics in QDs, especially in exciton decay, ${ }^{8}$ exciton cooling, ${ }^{9-12}$ multi-exciton dynamics, ${ }^{13-16}$ and the formation of multi-excitons by carrier (exciton) multiplication $(\mathrm{CM}) \cdot{ }^{17-22}$ The latter process is particularly interesting for potential applications of QDs in the field of solar energy conversion. ${ }^{23}$ In a conventional silicon solar cell, much of the energy toward the UV end of the solar spectrum is lost through the generation of heat because of the very fast intraband carrier cooling after photogeneration..$^{23}$ A possible approach to harvest high-energy photons more efficiently would be to utilize $\mathrm{CM}$. This mechanism involves the generation of more than one exciton from a single high-energy photon with high efficiency (also termed "multiple exciton generation"). Hence, the excess (i.e., above band gap) energy of a high-energy photon is converted into an additional exciton instead of being converted into heat. Although the $\mathrm{CM}$ process is inefficient in bulk materials, it has recently been demonstrated that $\mathrm{CM}$ can occur with high efficiency in

* Corresponding author. Telephone: +31-20-6081234. Fax: +31-206684106. E-mail: j.pijpers@amolf.nl.

FOM Institute for Atomic and Molecular Physics.

$\doteqdot$ Utrecht University.

$\S$ Hebrew University of Jerusalem.
QD materials ${ }^{17-22}$ with a demonstrated yield of up to seven carriers per photon. ${ }^{20}$

As in bulk materials, the presence of dopants has a large effect on the electronic properties of QDs. For example, the conductivity of nanocrystalline CdSe solids increases by many orders of magnitude as a result of $n$-type doping. ${ }^{24-26}$ Dopants may also affect other properties of QDs, such as multi-exciton dynamics. Therefore, knowledge on the effects of dopants on, for instance, $\mathrm{CM}$ in QD materials is important for the design of photovoltaic devices. The effect of the presence of dopants on the CM efficiency has not been reported before.

In this article, we investigate multi-exciton dynamics and $\mathrm{CM}$ in colloidal InAs/CdSe/ZnSe core-shell QDs. ${ }^{1}$ To our knowledge, this is the first such study in colloidal InAs QDs. InAs has the special characteristic that the hole band mass $m_{\mathrm{h}}$ is much larger than the electron mass $m_{\mathrm{e}}\left(m_{\mathrm{h}} / m_{\mathrm{e}}=17\right) .{ }^{27}$ This results in closely spaced energy levels in the valence band $(\sim 20 \mathrm{meV}$ for QDs with a $4.4 \mathrm{~nm}$ diameter) but widely spaced energy levels in the conduction band ( $\sim 300 \mathrm{meV}$ for QDs with a $4.4 \mathrm{~nm}$ diameter). ${ }^{28,29}$ The expectation that the CM efficiency is high in InAs QDs, because of the significantly different electron and hole masses, ${ }^{30}$ is corroborated by our results. Our approach combines time-resolved terahertz time-domain spectroscopy (THz-TDS), ${ }^{31}$ femtosecond transient absorption (TA), and quasicontinuous wave (qCW) excitation spectroscopy. ${ }^{32}$ For highenergy photons ( $>2$ times the band gap energy), $\mathrm{CM}$ results in multi-excitons, that is, $>1$ exciton per QD, which decay within tens of picoseconds. The presence of an exciton in a QD (generated by photodoping) greatly reduces the CM efficiency upon excitation with high-energy photons. We discuss the implications of our findings for the applications of InAs QDs as light absorbers in solar cells. 


\section{Experimental Methods}

Samples. In the experiments, we use high quality colloidal InAs/CdSe/ZnSe core/shell-1/shell-2 QDs, synthesized as reported elsewhere. ${ }^{1,33}$ The investigated QDs have an InAs core of 4.4-5.7 $\mathrm{nm}$ diameter (14\% size dispersion) onto which one atomic layer of $\mathrm{CdSe}$ and four layers of $\mathrm{ZnSe}$ are deposited. Samples are prepared by dissolving the QDs in toluene in a 1 $\mathrm{mm}$ thick cuvette. The core/shell structure leads to a significantly improved fluorescence quantum yield $(\sim 50 \%)^{1}$ as compared to QDs that are passivated with organic molecules like trioctylphosphine (2.5\%). ${ }^{33}$ This increase in the quantum yield is caused by the effective removal of surface defects by the inorganic shell material. Hereby, fewer carriers are trapped at the surface, and emission quenching via nonradiative decay channels is suppressed.

Time-Resolved Spectroscopy. Before investigating CM, it is important to understand multi-exciton recombination dynamics and spectroscopy of InAs QDs. In the time-resolved experiments, we generate multi-excitons in $4.4 \mathrm{~nm}$ QDs by sequential absorption of $800 \mathrm{~nm}$ photons and monitor the recombination dynamics by THz-TDS and TA. THz-TDS has previously been successfully applied to probe the transient hole populations in QDs. ${ }^{12,34}$ This technique uses a weak electromagnetic field $(\sim 1 \mathrm{kV} / \mathrm{cm})$ to probe the sample response at low energies $(\sim 4 \mathrm{meV})$ following photoexcitation with $150 \mathrm{fs}, 800$ $\mathrm{nm}$ laser pulses. The low-energy $\mathrm{THz}$ photons of our probe mean that the differential, pump-induced change in the THz transmission, $\Delta E_{\mathrm{THz}}$, is proportional to the population of the lowest energy hole state as a function of time $\tau$ after excitation (see refs 12 and 34). In addition, in order to study electron dynamics, femtosecond TA measurements are performed on a different setup. After photoexcitation of our QDs with $50 \mathrm{fs}$ laser pulses (wavelength $800 \mathrm{~nm}$ ), the bleach and the stimulated emission of the lowest energy inter-band transition (i.e., the absorption generating an electron and a hole in their respective lowest energy states, described by $\Delta \alpha_{1 S}$ ) are probed with a second 65 fs pulse (wavelength $1200 \mathrm{~nm}$ ) at time $\tau$ after photoexcitation. Since this transition requires the electron and hole to be in their lowest energy states, the TA measurements provide complementary information about electron dynamics. ${ }^{12}$

THz-TDS was also used to study CM. The number of excitons per QD $\left(N_{x}\right)$ immediately following photoexcitation were determined by normalizing the THz-TDS signal at the peak just after time zero by the value of the signal at $\tau=100 \mathrm{ps}$ after both tri-exciton decay and bi-exciton decay are complete. ${ }^{35}$ This procedure was followed at various 400 and $800 \mathrm{~nm}$ fluences. The effect of photodoping the QDs on CM has also been investigated using THz-TDS. Prior $(\sim 10 \mathrm{~ns})$ to exciting the QDs with a $400 \mathrm{~nm}$ laser pulse, the sample is illuminated with an intense $800 \mathrm{~nm} 150 \mathrm{fs}$ pulse $(1.5 \mathrm{~mJ} /$ pulse $)$. We confirmed that this pre-excitation leads to a complete saturation of the QDs with single excitons, as generated multi-excitons will decay to a single exciton state before the arrival of the subsequent 400 $\mathrm{nm}$ pulse. The $400 \mathrm{~nm}$ pulse (low fluence, $\sim 0.28 \mathrm{~J} / \mathrm{m}^{2}$ ) arrives at $\tau \sim 0$ ps on the sample, leading to the generation of multiexcitons by $\mathrm{CM}$. The $\mathrm{THz}$ signal is detected as a function of time after the $400 \mathrm{~nm}$ excitation while chopping the $800 \mathrm{~nm}$ pulse; that is, we determine how the CM efficiency changes as a result of photodoping by the $800 \mathrm{~nm}$ pulse. In this manner, the effect of the presence of single excitons on the CM efficiency could be determined.

Quasi-Continuous Wave Spectroscopy. The time-resolved data are complemented by the multi-exciton spectroscopy performed using the $\mathrm{qCW}$ excitation method. In this method, the QDs are excited by long ( $5 \mathrm{~ns}$ ) optical excitation pulses (532 and $1064 \mathrm{~nm}$ ) where the photon absorption rate depends on the intensity of the pulse. When the photon absorption rate exceeds the Auger recombination rate, a significant population of multiply excited QDs is generated, which is reflected in the transient emission spectra as measured at the peak of the excitation pulse. By multiple peak fittings of these transient spectra, it is possible to extract the spectral location of the multiexcitonic emission peaks relative to the exciton peak, providing, in this case, information about the bi- and tri-exciton binding energies. A detailed description of the method and the experimental setup can be found elsewhere. ${ }^{32}$

The $\mathrm{qCW}$ spectroscopy was also used to investigate CM, this time on the basis of differences in the emission spectra rather than by absorption. Briefly, this method relies on measuring the ratio of the tri-exciton emission to the bi-exciton emission at constant photon absorption rates for different excitation wavelengths (above and below the CM threshold). The measurement is performed in the intensity regime of the transient state filling in which the QDs are already pre-excited with a single exciton, similar to the THz-TDS photodoping experiment. As the tri-exciton and bi-exciton emission peaks are wellseparated spectrally, we can measure their intensity ratio reliably. The relative increase in this ratio, which is observed for abovethreshold excitation wavelengths compared to those at belowthreshold, is a measure of the CM efficiency.

\section{Results and Discussion}

Multi-Exciton Dynamics and Spectroscopy. We begin our study by measuring the dynamics and spectroscopy of multiexcitons in the InAs QDs. We first discuss the time-resolved data, followed by the qCW results. Figure 1 shows the $1 \mathrm{~S} \mathrm{TA}$ dynamics and the $\mathrm{THz}$ dynamics for a range of $800 \mathrm{~nm}$ excitation intensities. At low excitation fluences and low photon energies, we expect no more than one exciton per excited QD to be generated. Indeed, for the lowest $800 \mathrm{~nm}$ fluences investigated (see orange traces in Figure 1a,b), we observe a steplike signal which persists even on nanosecond time scales, in agreement with the very long (170-300 ns) radiative lifetime of the single exciton state. The initial fast rise in signal may be attributed to the rapid (subpicosecond) cooling of excited electrons and holes to their respective lowest energy states. ${ }^{9-12}$ From the TA dynamics of Figure 1a, it can therefore be concluded that, at fluences $\leq 0.44 \mathrm{~J} / \mathrm{m}^{2}$, single excitons are present in the QDs.

On increasing the excitation fluence, however, we observe faster decay components in both the TA traces and the THzTDS traces (see Figure 1), which we attribute to the decay of multi-excitons (i.e., the presence of $>1$ exciton per QD). For a fluence of $\sim 1 \mathrm{~J} / \mathrm{m}^{2}$, we observe an exponential decay with a time constant of 30 ps to a long-time signal plateau. This time constant is assigned to the decay of bi-excitons created by the multiphoton absorption of the pump. The time scale is much faster than bi-exciton decay dynamics in epitaxial QDs where decay constants on the order of nanoseconds have been found. ${ }^{36}$ This slower decay can be understood by noting the larger size ( $\sim 49 \mathrm{~nm}$ radius) of epitaxial QDs, which will lead to weaker carrier-carrier interactions than in the considerably smaller (4.4 $\mathrm{nm}$ ) colloidal QDs under investigation here. It has been proposed that it is these carrier-carrier interactions that allow fast decay of multi-exciton states in small QDs through (nonradiative) Auger recombination. ${ }^{13,30}$ Indeed, the decay of 30 ps observed in Figure 1 is remarkably similar to bi-exciton decay dynamics 

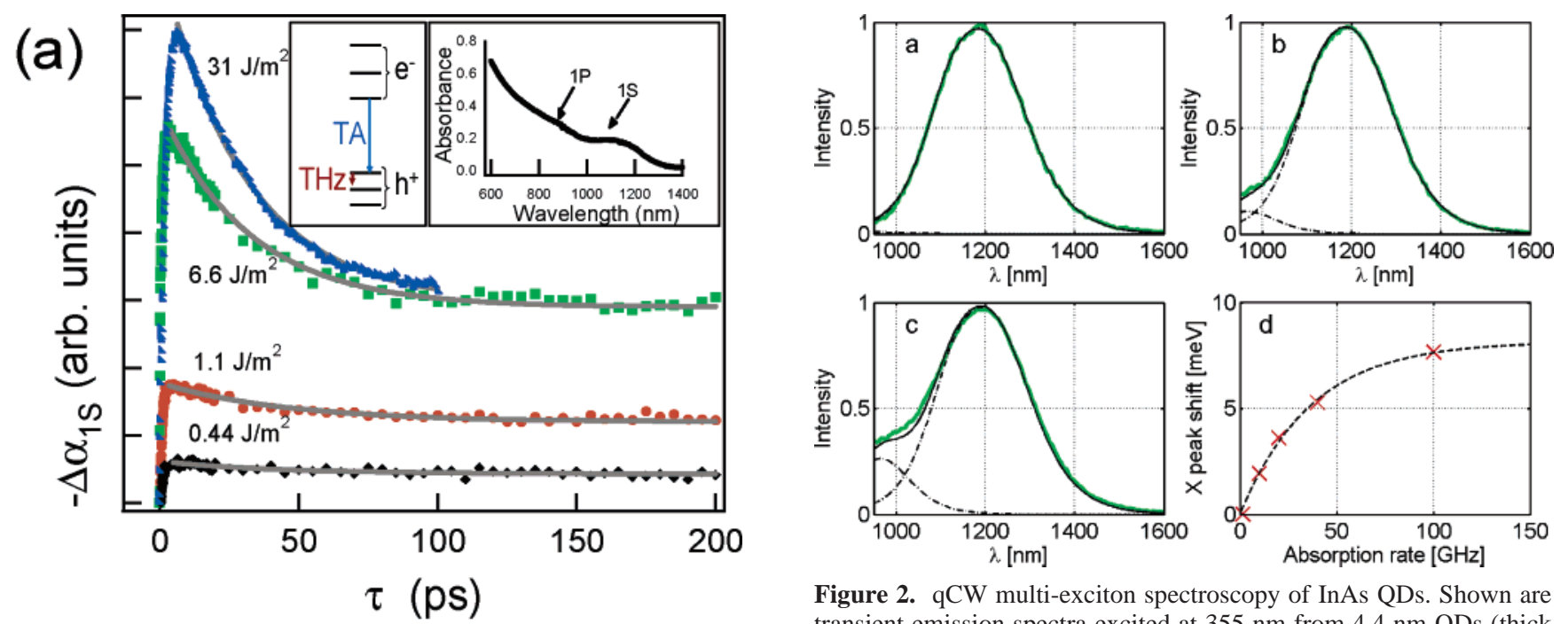

Figure 2. qCW multi-exciton spectroscopy of InAs QDs. Shown are transient emission spectra excited at $355 \mathrm{~nm}$ from $4.4 \mathrm{~nm}$ QDs (thick green lines) as well as their decomposition to the exciton/bi-exciton emission (centered, for low intensities at $1184 \mathrm{~nm}$, and red-shifted according to the fit results for higher fluences) and tri-exciton emission (centered at $965 \mathrm{~nm}$ ). The fit result is denoted by a thin black line. (a) Absorption rate of $1 \mathrm{GHz}$, practically no state filling is observed. (b) Absorption rate of $20 \mathrm{GHz}$, a redshift of $4.1 \mathrm{~nm}$ is observed along with some tri-exciton emission. (c) Absorption rate of $100 \mathrm{GHz}$, a redshift of $8.6 \mathrm{~nm}$ is observed along with an increased tri-exciton emission. (d) The measured exciton peak redshift as a function of the absorption rate, exhibiting saturation (as shown by the fitted dashed line) corresponding to a bi-exciton binding energy of about $8 \mathrm{meV}$.

In contrast to the TA measurements, the data from the $\mathrm{THz}$ decay dynamics can be used to extract multi-exciton decay rates. This is confirmed by Figure $1 \mathrm{~b}$ : at low fluences $\left(0.88 \mathrm{~J} / \mathrm{m}^{2}\right)$, only single excitons are present in the QDs, as evidenced by the absence of a significant decay of the signal within our time frame. At higher fluences $\left(2.54 \mathrm{~J} / \mathrm{m}^{2}\right)$, a bi-excitonic decay of $30 \mathrm{ps}$ is observed, which is in excellent agreement with the TA results from Figure 1a. Upon further increasing of the excitation fluence $\left(28.2 \mathrm{~J} / \mathrm{m}^{2}\right)$, even faster decay components are observed which can be attributed to tri-exciton decay. ${ }^{13}$ This data is wellfit by a double exponential decay with time constants of $3.5 \mathrm{ps}$ (tri-exciton decay) and $30 \mathrm{ps}$ (bi-exciton decay). The inset in Figure 1b shows the peak signal (immediately following photoexcitation) normalized to the long time signal (at $200 \mathrm{ps,}$ corresponding to 1 exciton per QD), $N_{x}$, as a function of fluence. This quantity is proportional to the number of detected excitons per QD (i.e., those which we are sensitive to with either TA or THz-TDS) immediately following photoexcitation. It can be seen that $N_{x}$ increases more in the $\mathrm{THz}$ data compared with the TA data, which saturates at $\sim 2$. This saturation illustrates that, probing the $1 \mathrm{~S}$ interband transition with $\mathrm{TA}$, one can effectively detect only the single and bi-exciton population dynamics because of the twofold degeneracy of the lowest electron level as discussed above. THz-TDS, meanwhile, can monitor the population dynamics of multiple $(>2)$ excitons since the degeneracy of the lowest energy hole levels is high and could not be accessed for the excitation fluences applied here.

To complement the time-resolved data, we perform multiexciton spectroscopy using the $\mathrm{qCW}$ excitation method. Transient spectra measured from QDs at several excitation rates are shown as thick green lines in Figure $2 \mathrm{a}-\mathrm{c}$. As the excitation rate is increased (by increasing the excitation intensity), the photon absorption rate increases, and state filling occurs, giving rise to two phenomena: a small redshift of the main peak due to the increasing contribution of the bi-exciton emission and 

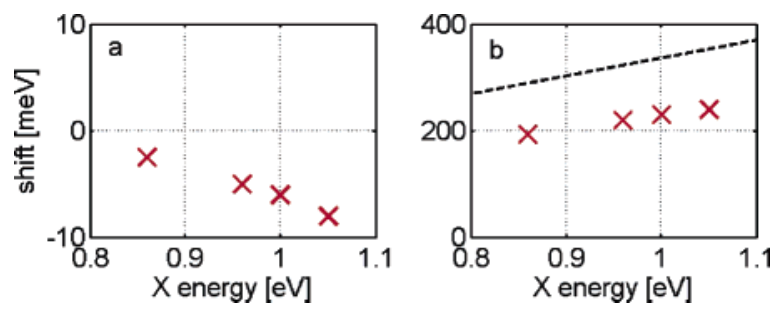

Figure 3. (a) Bi-exciton binding energy as a function of the band gap energy. (b) tri-exciton shift as a function of the band gap energy, along with the $1 \mathrm{~S}-1 \mathrm{P}$ energy separation from optical and STM data. ${ }^{37}$

the appearance of a blue-shifted shoulder due to tri-exciton emission.

The qCW spectra are analyzed in the following fashion: the single exciton emission is extracted from the emission data at a low excitation rate. All spectra at higher fluences are globally fitted using two peaks, one corresponding to the exciton/biexciton and the other to the tri-exciton. The individual peaks from the fit results are shown in Figure $2 a-c$ as dash-dotted lines, and their sum is plotted as a thin black line. To account for the bi-exciton binding energy, we allow in the fitting procedure for an independent redshift of the exciton/bi-exciton peak for each excitation rate, while keeping the line shape unchanged. At low excitation fluence, this shift is obviously zero, whereas at high fluences (where practically all of the transient emission is due to multi-exciton emission, since all dots are at least doubly excited), it saturates to a value corresponding to the bi-exciton binding energy. The measured redshift is shown in Figure $2 \mathrm{~d}$ as a function of excitation fluence for the $4.4 \mathrm{~nm}$ QDs ( $\times$ signs), along with a saturation curve fit (dashed line) of the form $\Delta E=\Delta_{\mathrm{Xx}}\left[1-\exp \left(-R / R_{0}\right)\right]$, where $R$ is the photon absorption rate. From the fit results, we conclude $\Delta_{\mathrm{XX}}$, the bi-exciton binding energy, to be about $8 \mathrm{meV}$. This procedure is repeated for several QD sizes, ranging from 4.4 to $5.7 \mathrm{~nm}$.

The extracted bi-exciton binding energies and tri-exciton shifts are shown in Figure 3 a,b, respectively. The bi-exciton binding energy in the InAs QDs is only a few milli-electronvolts (up to 10), significantly smaller than that observed in CdSe QDs of a similar size (in the range of $30 \mathrm{meV}$ ). ${ }^{38,39} \mathrm{~A}$ possible explanation for this difference may be the larger screening of charges in InAs because of the $40 \%$ larger dielectric constant compared with CdSe. Additional reasons for this difference may be the dense hole level structure in InAs QDs ${ }^{40}$ and the reduced overlap between the electron and the hole wavefunctions (because of the large difference in the effective mass). The tri-exciton shift is compared with the $1 \mathrm{~S}-1 \mathrm{P}$ energy difference as obtained from optical and scanning tunneling microscopy (STM) measurements ${ }^{37}$ and reflects a tri-exciton binding energy of the order of $100 \mathrm{meV}$, comparable to binding energies observed in $\mathrm{CdSe}$ QDs. ${ }^{39}$ Below, we will use the tri-exciton emission peak to quantify CM in InAs QDs.

Carrier Multiplication. With this information on multiexciton spectroscopy and dynamics, we proceed with investigating the CM process in the InAs dots. Multi-exciton generation through $\mathrm{CM}$ rather than through multiphoton absorption may be of interest for solar cell applications. ${ }^{23}$ In this process, multiexcitons are generated through the conversion of a single highenergy photon into multiple excitons. Several mechanisms have been proposed in the literature to explain CM, including impact ionization (II), ${ }^{17,41,42}$ the coherent superposition of single and multi-exciton wavefunctions, ${ }^{18}$ and the occurrence of virtual single exciton states. ${ }^{19,30}$ Although the CM mechanism is still under debate, recent publications have shown that II can explain

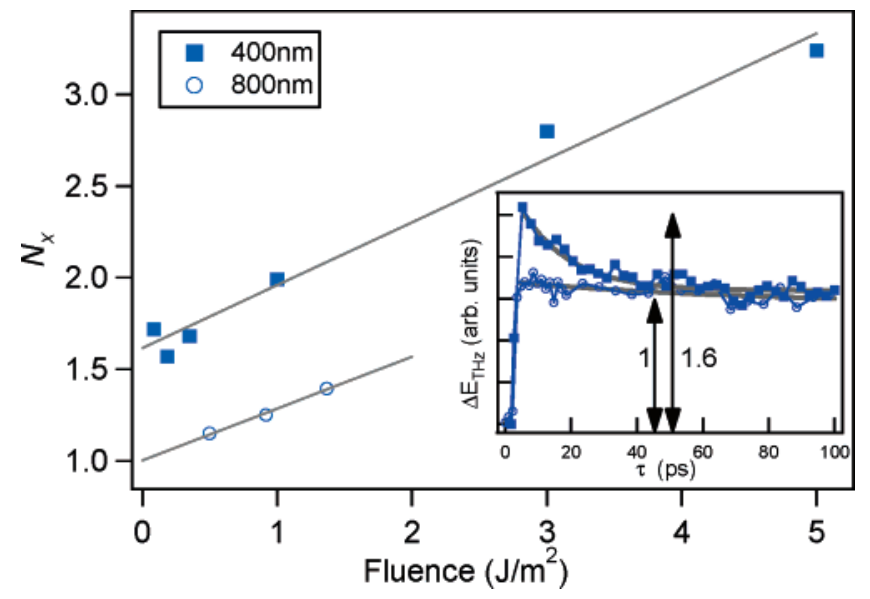

Figure 4. Number of excitons per QD as a function of the excitation fluence (400 and $800 \mathrm{~nm}$ ). The number of excitons per QD was determined by dividing the signal at $t_{0}$ by the value of the signal at $\tau$ $=100 \mathrm{ps}$. The inset shows exciton decay dynamics at the 400 and 800 $\mathrm{nm}$ excitation fluence, for identical products of absorption cross section and fluence. For these traces, the fluences were chosen sufficiently low to ensure that less than one photon was absorbed per QD, as is evident from the $800 \mathrm{~nm}$ data.

most experimental observations. ${ }^{41,42}$ With our experimental techniques, we can observe $\mathrm{CM}$, but we cannot investigate the $\mathrm{CM}$ mechanism because either we do not have sufficient time resolution $(\mathrm{qCW})$ or we do not have the ability to vary the excitation energy over a broad range (THz-TDS).

In any case, it has been argued that the activation threshold for $\mathrm{CM}$ is expected to be relatively low in InAs QDs because of effective mass arguments. ${ }^{30,42}$ In InAs, the band mass of the holes is significantly higher than the electron mass $\left(m_{\mathrm{h}}=0.41\right.$ and $\left.m_{\mathrm{e}}=0.024\right)^{1}$ resulting in an asymmetric density of states in the valence and conduction band. According to ref 21, the minimum photon energy to produce $\mathrm{CM}\left(\hbar \omega_{\mathrm{CM}}\right)$ is approximately given by

$$
\hbar \omega_{\mathrm{CM}}=\left(2+m_{\mathrm{e}} / m_{\mathrm{h}}\right) E_{\mathrm{bg}}
$$

where $E_{\mathrm{bg}}$ is the QD energy gap. For the $4.4 \mathrm{~nm}$ InAs core QDs $\left(E_{\mathrm{bg}}=1.13 \mathrm{eV}\right.$, see inset Figure 1a), the minimum photon energy for $\mathrm{CM}$ is calculated using eq 1 to be $2.31 \mathrm{eV}$. Hence, one would expect $400 \mathrm{~nm}$ (3.1 eV) photons to readily induce CM.

Figure 4 shows the number of excitons per QD $\left(N_{x}\right)$ immediately following photoexcitation measured with $\mathrm{THz}-$ TDS. For both $800 \mathrm{~nm}$ and $400 \mathrm{~nm}$ excitation, $N_{x}$ increases as a function of fluence because of multiphoton absorption. By extrapolating to zero fluence (lines in Figure 4), a maximum of one photon is absorbed per QD, $N_{x}=1$ for $800 \mathrm{~nm}$ excitation, while, in the case of comparable $400 \mathrm{~nm}$ excitation fluences, an average of 1.6 excitons per QD are generated. The CM factor of 1.6 is also evident from the raw data depicted in the inset of Figure 4, which depicts 800 and $400 \mathrm{~nm}$ data for identical products of the fluence and absorption cross section.

The fact that $N_{x}<2$ for the lowest $400 \mathrm{~nm}$ fluences indicates that not every absorbed $400 \mathrm{~nm}$ photon results in the formation of multi-excitons. This observation is consistent with refs $18-$ 22 , where a strong increase of the $\mathrm{CM}$ efficiency as a function of the ratio of the photon and band gap energies $\left(E_{\mathrm{ph}} / E_{\mathrm{bg}}\right)$ is observed. Schaller et al. report that this increase follows a linear relationship (with a slope of 1.13) for both $\mathrm{CdSe}$ and $\mathrm{PbSe}$, in spite of the different crystal structures of these materials. Since we only excite with one photon energy $\left(E_{\mathrm{ph}} / E_{\mathrm{bg}}=2.74\right)$, we 


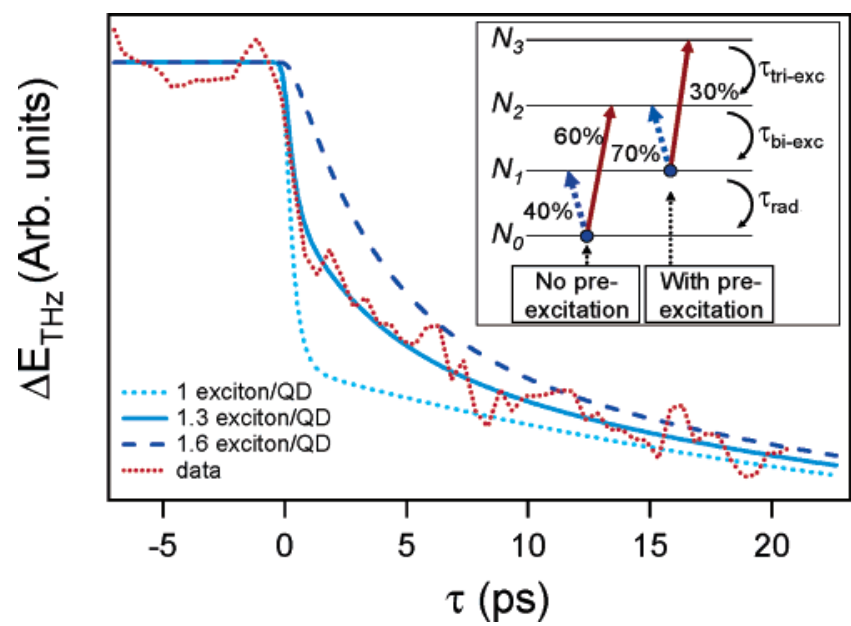

Figure 5. Differential signal of the exciton induced $\mathrm{THz}$ modulation, while chopping the $800 \mathrm{~nm}$ pre-excitation pulse. The steep decrease of the signal right after $\tau=0 \mathrm{ps}$ is an indication that the CM efficiency changes as a result of the presence of single excitons. The data can be described by a four-level model (inset), which is described in the text. The model describes the dynamics optimally for a CM efficiency of $130 \%$ (blue solid curve), which suggests that, on average, 1.3 excitons per absorbed photon are generated as opposed to 1.6 for the QD without pre-excitation. The inset gives a schematic representation of the model, as described in the text.

cannot confirm this dependence in InAs. However, if we extrapolate from the point of $160 \% \mathrm{CM}$ efficiency at $E_{\mathrm{ph}} / E_{\mathrm{bg}}=$ 2.74 using a slope of $113 \%$ per $E_{\mathrm{g}}$, we predict that the onset for CM in InAs QDs lies at $2.14 E_{\mathrm{g}}$. This value is very close to the predicted threshold value of $2.05 E_{\mathrm{g}}$ according to eq 1 .

To go beyond the more conventional one photon to two excitons CM process, we also measured the effect of (photo)doping the QDs on the CM efficiency. This was performed by pre-exciting the QDs at $800 \mathrm{~nm}$ to generate a single exciton population, as explained in the experimental section, followed by excitation at $400 \mathrm{~nm}$. The differential signal (i.e., the difference in the $\mathrm{THz}$ signal with and without $800 \mathrm{~nm}$ pre-excitation) is given in Figure 5. It can be seen that there is a very fast decrease of the signal at $\tau=0 \mathrm{ps}$. This decrease indicates directly that photodoping by the $800 \mathrm{~nm}$ pulse leads to fewer excitons immediately after photoexcitation by the 400 $\mathrm{nm}$ pulse, which indicates that the CM efficiency decreases in the presence of an exciton.

The change in CM efficiency can be quantified by a fourlevel model that describes the system: the four levels consist of $N_{0}, N_{1}, N_{2}$, and $N_{3}$ containing zero, one, two, and three excitons per QD, respectively. This four-level system is depicted schematically in the inset in Figure 5. Decay between the different states is indicated by the curved arrows, with time constants discussed in the text above.

In the case of no pre-excitation, the $\mathrm{CM}$ factor of 1.6 dictates that $60 \%$ of the QDs are excited from the ground state into $\mathrm{N}_{2}$ via $\mathrm{CM}$ after a $400 \mathrm{~nm}$ photon is absorbed. The remaining $40 \%$ of the QDs do not undergo CM and are excited into $N_{1}$. In the case of pre-excitation with $800 \mathrm{~nm}$ light, all QDs are in the $N_{1}$ state at $\tau=0 \mathrm{ps}$, when the $400 \mathrm{~nm}$ pulse arrives at the sample. In this situation, a certain fraction of the QDs undergoes CM and is excited from the $N_{1}$ level into $N_{3}$. The fraction of QDs that does not undergo CM is excited from the $N_{1}$ level into $N_{2}$. The blue curves in Figure 5 represent the situations where $0 \%$ (dotted, blue), 30\% (solid), and 60\% (dashed) of the QDs undergo CM. These situations are defined as 100\%, 130\%, and $160 \%$ CM efficiency, respectively. The model clearly describes the data optimally for a CM efficiency of $130 \pm 10 \%(1.3$ excitons per QD). As can be seen in Figure 5, a CM efficiency of $160 \%$ (the case assuming pre-excitation does not affect $\mathrm{CM}$ ) clearly does not coincide with the data, implying that the $\mathrm{CM}$ efficiency in InAs QDs is reduced because of the presence of excitons.

The fact that the lowest electron level is twofold degenerated in InAs QDs (evident from the TA results in Figure 1a) suggests that the CM efficiency should be lower in the presence of dopants, as CM will then be energetically less favorable. With an exciton already present in a QD, CM following absorption of a $400 \mathrm{~nm}$ photon in a $4.4 \mathrm{~nm}$ QD must result in the formation of one $1 \mathrm{~S}\left(E_{\mathrm{bg}}=1.13 \mathrm{eV}\right)$ and one $1 \mathrm{P}$ exciton $\left(E_{\mathrm{bg}}=1.43\right.$ $\mathrm{eV})$. An "effective band gap", $E_{\mathrm{bg}}^{\mathrm{eff}}$, for these excitons can be defined as the average energy, which is $1.28 \mathrm{eV}$ or possibly lower by up to $0.05 \mathrm{eV}$ because of the tri-exciton binding energy (Figure 3b). Hence, for constant photon energy, the photon energy relative to the effective band gap, $E_{\mathrm{ph}} / E_{\mathrm{bg}}$, for $\mathrm{CM}$ in the presence of an exciton will be smaller than that in the absence of one, and CM is expected to occur with lower probability. Following the calculations of Schaller et al. ${ }^{21}$ along the lines presented above, this should lead to a decrease of the $\mathrm{CM}$ efficiency to roughly $\sim 1.30$, in very good agreement with our measurements.

The reduction of the CM efficiency from 1.6 to 1.3 in the presence of an exciton is confirmed by qCW experiments. Since the $5 \mathrm{~ns}$ excitation pulse used in the $\mathrm{qCW}$ experiments is much shorter than the $1 \mathrm{~S}$ exciton radiative lifetime, the QD is always (at least) singly excited. To corroborate the $\mathrm{THz} \mathrm{CM}$ efficiencies, we perform a qCW experiment utilizing both $532 \mathrm{~nm}$ and 1064 $\mathrm{nm}$ excitations for the $5.7 \mathrm{~nm}$ dots $(0.85 \mathrm{eV}$ emission). The radius of the QDs in these experiments is such that the (fixed) fundamental $(1064 \mathrm{~nm})$ and second harmonic $(532 \mathrm{~nm})$ of the $\mathrm{Nd}$ :YAG laser used correspond to excess energies relative to the band gap which correspond roughly with those used in the smaller particles in the $\mathrm{THz}$ experiments with 400 and $800 \mathrm{~nm}$ excitation. As such, the two combinations of excitation wavelengths and QD size used in the $\mathrm{THz}$ and $\mathrm{qCW}$ measurements give similar predictions for the onset of $\mathrm{CM}$ according to eq 1 (discussed further below).

We follow the CM process by probing the emission rather than the absorption, introducing a new approach to study the $\mathrm{CM}$ process. To this end, transient emission spectra are obtained for both $1064 \mathrm{~nm}$ (CM not possible) and $532 \mathrm{~nm}$ (CM possible) excitation wavelengths at several pulse energies, as shown in Figure 6 (where red lines show $1064 \mathrm{~nm}$ excitation and green lines denote $532 \mathrm{~nm}$ excitation). These spectra are then decomposed to the exciton/bi-exciton component (which practically lie on top of each other as shown in Figure 2) and the tri-exciton component (as shown in the black lines for the highest energy $532 \mathrm{~nm}$ spectrum). The ratio between the two peaks is plotted in the inset as a function of the photon absorption rate. Without CM, the two lines should overlap, as the population in that case is strictly dependent on state filling following single excitation events. This was confirmed in experiments on $\mathrm{CdSe} / \mathrm{ZnS}$ dots using both $355 \mathrm{~nm}$ and $532 \mathrm{~nm}$ excitation. Here, however, we observe a clear shift between the two curves, where enhanced tri-exciton contribution is present upon $532 \mathrm{~nm}$ excitation. This excess tri-exciton emission can only be due to CM, leading to a larger number of carriers and hence an enhanced tri-exciton contribution at $532 \mathrm{~nm}$. This observation provides a unique signature for $\mathrm{CM}$ related to the enhanced emission of the higher exciton states.

The CM efficiency can be obtained from these experiments by the scaling factor of the $x$-axis compression required to 


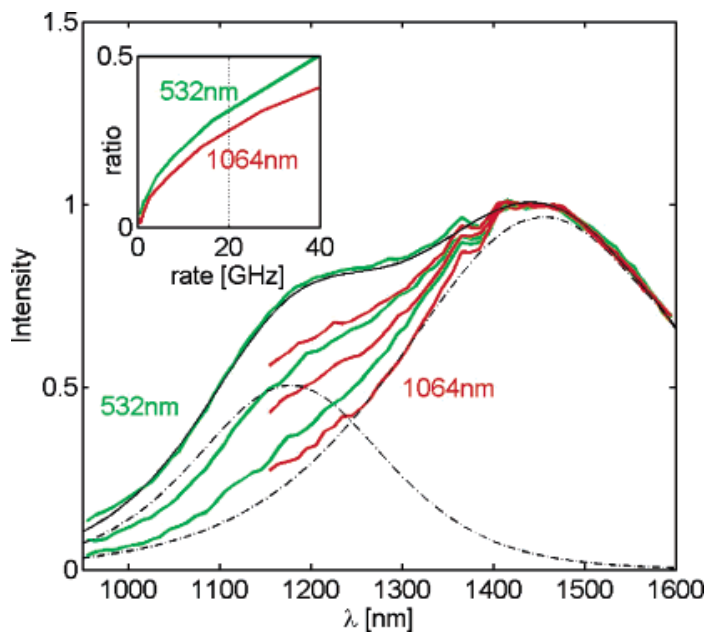

Figure 6. $\mathrm{CM}$ in a $\mathrm{qCW}$ configuration: Transient spectra at 3, 14, and $28 \mathrm{GHz}$ absorption rates with $1064 \mathrm{~nm}$ pumping (red lines) along with transient spectra at 4,16 , and $40 \mathrm{GHz}$ with $532 \mathrm{~nm}$ pumping (green lines). The decomposition of the $40 \mathrm{GHz}, 532 \mathrm{~nm}$ pumping emission into exciton/bi-exciton and tri-exciton peaks is shown in black. The inset shows the tri-exciton to exciton/bi-exciton intensity ratio as a function of the absorption rate. The larger ratio at $532 \mathrm{~nm}$ pumping (green line) is due to carrier CM. A CM efficiency of 1.4 is obtained.

overlap the two curves showing the emission ratio, in the region for photon absorption rates $1>\tau_{\text {bi-exciton }} R>0.2$ ( $R$ being the photon absorption rate). This assignment is supported by a modified ladder climbing model for the $\mathrm{qCW}$ experiment, ${ }^{32}$ taking into account the probability for $\mathrm{CM}$, that is, a direct transition from one to three excitons. Using this criterion, we obtain a factor of 1.4 corresponding to a CM efficiency of $140 \%$.

To quantitatively compare the $\mathrm{THz}$ results with the $\mathrm{qCW}$, we note the slight difference in the $E_{\mathrm{ph}} / E_{\mathrm{bg}}$ ratio in both experiments, which will obviously affect the CM efficiency. For the qCW experiment, exciting the $5.7 \mathrm{~nm}$ cores with 532 $\mathrm{nm}$ light corresponds to performing the experiment at $2.47 E_{\mathrm{g}}$. For the $\mathrm{THz}$ case, under $400 \mathrm{~nm}$ excitation and $4.4 \mathrm{~nm}$ cores, the QDs are excited at $2.42 E_{\mathrm{g}}$. Since the predicted onset for $\mathrm{CM}$ lies at $2.14 E_{\mathrm{g}}, \mathrm{CM}$ is expected to be slightly more efficient in the $\mathrm{qCW}$ experiment. Therefore, the qCW CM efficiency of $140 \%$ is in reasonable agreement with CM efficiency of $130 \%$ found from the THz-TDS data in Figure 5 and confirms the reduction of $\mathrm{CM}$ due to photodoping.

\section{Conclusion}

We have investigated ultrafast exciton dynamics and CM in colloidal InAs/CdSe/ZnSe core-shell QDs by combining THzTDS, femtosecond TA spectroscopy, and qCW excitation spectroscopy. The time constants of bi-exciton and tri-exciton recombination were found to be 30 and $3.5 \mathrm{ps,} \mathrm{respectively.}$ When exciting the QDs with high-energy photons, CM occurs with high efficiency. Photodoping the QDs leads to a decrease of the CM efficiency because of the twofold degeneracy of the lowest electron level.

The fact that the onset of CM occurs at a relatively low $E_{\mathrm{ph}} /$ $E_{\mathrm{bg}}$ ratio of 2.14 makes InAs QDs a very suitable material for photovoltaic applications. Since bi-exciton recombination occurs within tens of picoseconds, the extraction of bi-excitons from InAs QDs might be faster than exciton recombination processes. We further demonstrate that the presence of dopants leads to a decrease of the CM efficiency in InAs QDs. Therefore, doping of InAs QDs is not a logical strategy for an optimal solar cell design.
Acknowledgment. This work is part of the Joint Solar Programme (JSP) of the Stichting voor Fundamenteel Onderzoek der Materie FOM, which is supported financially by Nederlandse Organisatie voor Wetenschappelijk Onderzoek (NWO). The JSP is co-financed by gebied Chemische Wetenschappen of NWO and Stichting Shell Research. The work was partially supported by the Israel ministry of science under the "Tashtiot" program.

\section{References and Notes}

(1) Aharoni, A.; Mokari, T.; Popov, I.; Banin, U. J. Am. Chem. Soc. 2006, 128, 257.

(2) Bruchez, M.; Moronne, M.; Gin, P.; Weiss, S.; Alivisatos, A. P. Science 1998, 281, 2013.

(3) Chan, W. C. W.; Nie, S. Science 1998, 281, 2016.

(4) Malko, A. V.; Mikhailovsky, A. A.; Petruska, M. A.; Hollingsworth, J. A.; Htoon, H.; Bawendi, M. G.; Klimov, V. I. Appl. Phys. Lett. 2002, 81,1303 .

(5) Tessler, N.; Medvedev, V.; Kazes, M.; Kan, S.-H.; Banin, U. Science 2002, 295, 1506.

(6) Steckel, J. S.; Coe-Sullivan, S.; Bulovic, V.; Bawendi, M. G. Adv. Mater. 2003, 25, 1862.

(7) Olsson, Y. K.; Chen, G.; Rapaport, R.; Fuchs, D. T.; Sundar, V. C.; Steckel, J. S.; Bawendi, M. G.; Aharoni, A.; Banin, U. Appl. Phys. Lett. 2004, 85, 4469.

(8) van Driel, A. F.; Allan, G.; Delerue, C.; Lodahl, P.; Vos, W. L.; Vanmaekelbergh, D. Phys. Rev. Lett. 2005, 95, 236804.

(9) Klimov, V. I.; McBranch, D. W. Phys. Rev. Lett. 1998, 80, 4028.

(10) Klimov, V. I.; McBranch, D. W.; Leatherdale, C. A.; Bawendi, M. G. Phys. Rev. B: Condens. Matter Mater. Phys. 1999, 60, 13740.

(11) Guyot-Sionnest, P.; Shim, M.; Matranga, C.; Hines, M. Phys. Rev. B: Condens. Matter Mater. Phys. 1999, 60, 2181.

(12) Hendry, E.; Koeberg, M.; Wang, F.; Zhang, H.; de Mello Donegá, C.; Vanmaekelbergh, D.; Bonn, M. Phys. Rev. Lett. 2006, 96, 057408.

(13) Klimov, V. I.; Mikhailovsky, A. A.; McBranch, D. W.; Leatherdale, C. A.; Bawendi, M. G. Science 2000, 287, 1011.

(14) Achermann, M.; Hollingsworth, J. A.; Klimov, V. I. Phys. Rev. B: Condens. Matter Mater. Phys. 2003, 68, 245302.

(15) Fisher, B.; Caruge, J.-M.; Chan, Y.-T.; Halpert, J.; Bawendi, M. G. Chem. Phys. 2005, 318, 71 .

(16) Bonati, C.; Mohamed, M. B.; Tonti, D.; Zgrablic, G.; Haacke, S.; van Mourik, F.; Chergui, M. Phys. Rev. B: Condens. Matter Mater. Phys. 2005, 71, 205317.

(17) Schaller, R. D.; Klimov, V. I. Phys. Rev. Lett. 2004, 92, 186601. (18) Ellingson, R. J.; Beard, M. C.; Johnson, J. C.; Yu, P.; Micic, O. I.; Nozik, A. J.; Shabaev, A.; Efros, A. L. Nano Lett. 2005, 5, 865.

(19) Schaller, R. D.; Agranovich, V. M.; Klimov, V. I. Nat. Phys. 2005 $1,189$.

(20) Schaller, R. D.; Sykora, M.; Pietryga, J. M.; Klimov, V. I. Nano Lett. 2005, 6, 424.

(21) Schaller, R. D.; Petruska, M. A.; Klimov, V. I. Appl. Phys. Lett. 2005, 87, 253102.

(22) Murphy, J. E.; Beard, M. C.; Norman, A. G.; Ahrenkiel, S. P.; Johnson, J. C.; Yu, P.; Mićić, O. I.; Ellingson, R. J.; Nozik, A. J. J. Am. Chem. Soc. 2006, 128, 3241.

(23) Nozik, A. J. Physica E (Amsterdam, Neth.) 2002, 14, 115.

(24) Roest, A. L.; Kelly, J. J.; Vanmaekelbergh, D. Phys. Rev. Lett. 2002, 89, 036801 .

(25) Yu, D.; Wang, C.; Guyot-Sionnest, P. Science 2003, 300, 1277. (26) Houtepen, A. J.; Vanmaekelbergh, D. J. Phys. Chem. B 2005, 109, 19634

(27) Madelung, O.; Schulz, M.; Heiss, H. Numerical Data and Functional Relationships in Science and Technology, New Series; SpringerVerlag: New York, 1982; Vol. 17.

(28) Krapf, D.; Kan, S.-H.; Banin, U.; Millo, O.; Sa'ar, A. Phys. Rev. B: Condens. Matter Mater. Phys. 2004, 69, 073301.

(29) Woggon, U. Optical Properties of Semiconductor Quantum Dots; Springer-Verlag: Berlin-Heidelberg, Germany, 1997.

(30) Klimov, V. I. J. Phys. Chem. B 2006, 110, 16827.

(31) Beard, M. C.; Turner, G. M.; Schmuttenmaer, C. A. J. Phys. Chem. B 2002, 106, 7146.

(32) Oron, D.; Kazes, M.; Shweky, I.; Banin, U. Phys. Rev. B: Condens. Matter Mater. Phys. 2006, 74, 115333.

(33) Guzelian, A. A.; Banin, U.; Kadavanich, A. V.; Peng, X.; Alivisatos, A. P. Appl. Phys. Lett. 1996, 69, 1432.

(34) Wang, F.; Shan, J.; Islam, M. A.; Herman, I. P.; Bonn, M.; Heinz, T. F. Nat. Mater. 2006, 5, 861 .

(35) Schaller, R. D.; Klimov, V. I. Phys. Rev. Lett. 2006, 96, 097402.

(36) Kono, S.; Kirihara, A.; Tomita, A.; Nakamura, K.; Fujikata, J.; Ohashi, K.; Saito, H.; Nishi, K. Phys. Rev. B: Condens. Matter Mater. Phys. 2005, 72, 155307. 
(37) Banin, U.; Cao, Y.; Katz, D.; Millo, O. Nature 1999, 400, 542.

(38) Achermann, M.; Hollingsworth, J. A.; Klimov, V. I. Phys. Rev. B: Condens. Matter Mater. Phys. 2003, 68, 245302.

(39) Bonati, C.; Mohamed, M. B.; Tonti, D.; Zgrablic, G.; Haacke, S.; van Mourik, F.; Chergui, M. Phys. Rev. B: Condens. Matter Mater. Phys. 2005, 71, 205317.
(40) Zhu, Y. H.; Zhang, X. W.; Xia, J. B. Phys. Rev. B: Condens. Matter Mater. Phys. 2006, 73, 165326.

(41) Allan, G.; Delerue, C. Phys. Rev. B: Condens. Matter Mater. Phys. 2006, 73, 205423.

(42) Franceschetti, A.; An, J. M.; Zunger, A. Nano Lett. 2006, 6, 2191. 